\title{
Multiple Types of Human Papillomavirus in Cervical Samples in Women in Campo Grande, MS, Brazil
}

\author{
Inês Aparecida Tozetti ${ }^{1}$, Ilzia Doraci Lins Scapulatempo ${ }^{1,2}$, \\ Vicky Liege Kawski ${ }^{3}$, Antonio Walter Ferreira ${ }^{4}$ and \\ José Eduardo Levi ${ }^{5}$
}

\author{
${ }^{1}$ Pathology Department of the Federal University of \\ Mato Grosso do Sul (UFMS); ${ }^{2}$ Cancer Prevention \\ Center; ${ }^{3}$ Laboratory of Immunology - UFMS, Campo \\ Grande, MS; 4 - Biolab-Merieux S/A and Tropical \\ Medicine Institute of São Paulo (IMT-SP), 5 - \\ Laboratory of Virology of IMT-SP, São Paulo, SP, Brazil
}

\begin{abstract}
We analyzed 87 cervical samples from Campo Grande, Mato Grosso do Sul, with a PGMY/GP ${ }^{+}$ nested PCR system. Positive samples were typed using E7 type-specific primer pairs for HPV 6/11, 16, 18, 45 and 66. Eighteen samples (22\%) were infected with HPV6/11, 18 samples (22\%) with HPV66, 13 samples $(15.9 \%)$ with HPV45, 8 samples $(9.8 \%)$ with HPV18 and 7 samples $(8.5 \%)$ with HPV16. Seventeen samples $(20.7 \%)$ were infected by two HPV types, and five samples $(6.1 \%)$ by three HPV types. We conclude that infection with multiple types is present at a high frequency in our population and that there is a relation between some types and cytological finds.

Key Words: Human papillomavirus, multiple types, nested PCR, TS-PCR.
\end{abstract}

Several molecular techniques have been used to detect HPV in cervical samples, including Hybrid Capture II (HCII) assay and the Polymerase Chain Reaction (PCR). The most common primers used in PCR to detect genital HPV are PGMY09/11 and $\mathrm{GP5}^{+} / 6^{+}$[1-3]. Fuessel Haws et al. found that PGMY/GP+ nested PCR detected more HPV types than did MY/GP+ nested PCR [4].

The determination of the HPV types may be done by several methods including Type-Specific PCR (TS-PCR) and Restriction Fragment Length Polymorphisms (RFLP). The latter relies on distinct patterns of amplicon digestion with seven restriction enzymes. However, these patterns may be difficult to discriminate, especially when clinical specimens contain two or more HPV types. Alternatively, PCR with type-specific primers targeting E6 and/or E7 non-conserved regions is a highly sensitive method that is easy to interpret and allows characterization of infections by multiple types [5-7]. Using E7-type specific-primer pairs for HPV 6/11, 16, 18, 45 and 66, we studied samples tested by a PGMY/GP+ nested-PCR system to precisely characterize HPV types and to identify multiply-infected patients.

Eighty-seven samples were collected from women, attended at the Centro de Prevenção ao Câncer, Campo Grande, Mato Grosso do Sul, Brazil. All patients had clinical suspision on HPV infection and came from public health service or medical clinics. Cytological diagnoses were reported using

Received on 22 June 2006; revised 27 September 2006.

Address for correspondence: Prof. Dr. Inês Aparecida Tozetti. Departamento de Patologia. Centro de Ciências Biológicas e da Saúde. Universidade Federal de Mato Grosso do Sul. Cidade Universitária s/n. Bairro Universitário. Campo Grande - MS. Caixa Postal 549, Zip Code: 79070-900. Brazil. E-mail: tozetti@nin.ufms.br Phone: (55-67) 3457388 .

The Brazilian Journal of Infectious Diseases 2006;10(5):309-310. (C) 2006 by The Brazilian Journal of Infectious Diseases and Contexto Publishing. All rights reserved. the 2001 Bethesda System [8]. The positive products from the PGMY/GP ${ }^{+}$nested PCR system were amplified using the techniques described by Walboomers et al. [9]; type-specific HPV primers were used, which amplify approximately $100 \mathrm{bp}$ in the E7 open reading frame of HPV 6/11, 16, 18, 45 and 66. Statistical analyses were performed using the Epinfo software, version 3.2, 2004 (CDC, Atlanta, Georgia).

Forty-two samples were typed by TS-PCR, and the distribution of HPV types was: 18 samples (22\%) were infected with HPV6/11, 18 samples (22\%) with HPV66, 13 samples (15.9\%) with HPV45, 8 samples $(9.8 \%)$ with HPV18 and 7 samples (8.5\%) with HPV16. Seventeen samples (20.7\%) were infected by two HPV types, and five samples $(6.1 \%)$ by three HPV types. In samples with atypical squamous cells of undetermined significance (ASCUS) cytology, a predominance of HPV $45(71 \%)$ was observed, and in samples with low grade squamous intraepithelial lesion (LSIL) cytology there was a predominance of HPV6/11 (50\%). High frequencies of HPV 16 $(31.3 \%)$ and HPV 18 (25\%) were observed in patients with LSIL cytology. Samples that were negative to intraepithelial lesion and malignancy (NILM) showed a high frequency of HPV6/11 (38.9\%) and HPV 66 (38.8\%). The sample that had high grade squamous intraepithelial lesion (HSIL) cytology was positive for HPV6/11 and HPV 66. The distribution of HPV 66 was similar among the ASCUS (43\%), LSIL (44\%) and NILM $(39 \%)$ cytological groups.

Among the patients, $26.8 \%$ were infected with multiple HPV types, similar to previous reports. Huang et al. studied patients with cervical carcinoma in China; they found $28.9 \%$ of the tumors to be infected with multiple HPV types [10]. Munoz et al. examined cases from several countries, and $13.9 \%$ of them were infected with multiple HPV types [11]. Eluf-Neto et al. found 5.1\% infection with multiple types in Brazil [12] and Levi et al. reported that $45 \%$ of the patients were infected with more than two HPV types, in a HIV-infected population from São Paulo, SP, Brazil [13]. Based on the wide range of 
these results, we suggest that geographical differences have an influence on the frequency of multiple HPV type infection.

Tawheed et al. found HPV66 in $2.5 \%$ of cervical lesions that had been diagnosed as high-grade cervical intraepithelial neoplasias, demonstrating a possible oncogenic potential of this HPV [14]. However, it has been classified as probably carcinogenic, an HPV that induces cervical intraepithelial neoplasia (CIN) I and II but rarely III $[11,15]$. Recently, the International Agency for Research on Cancer reassessed the carcinogenicity of HPV and classified HPV66 as carcinogenic for humans [16].

Munoz et al. reported $0.5 \%$ of the HPV66 among patients with invasive and in situ cervical cancer [11]; Poljak et al. reported $1.3 \%$ of this type in cervical specimens from women recognized as HPV-positive who were using an HCII high-risk cocktail [17]; Van Den Brule reported 2.7\% HPV66 in specimens derived from women participating in cervical cancer screening [18]; Speich et al. reported $4.0 \%$ of this type in cervical samples of West German women [19]. There have been reports of a high frequency of this type in an HIV-infected population $(9.4 \%$ and $20 \%)$ [13, 20]. However, Fuessel Haws et al. detected a higher frequency of HPV66 in samples positive for PGMY/ $\mathrm{GP}^{+}$nested PCR than in samples positive for $\mathrm{MY} / \mathrm{GP}^{+}$nested PCR ( $8.1 \%$ and $2.7 \%$ respectively) [4]. As we only typed

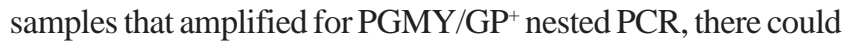
have been preferential selection for this HPV type. Improvement of the PGMY system compared to MY for HPV 66 amplification could explain our results.

Van den Brule et al. reported an association of HPV16 and HPV18 with CIN I/II and III [18]. They observed that low-risk (LR-HPV) oncogenic types were predominant in samples with LSIL/NILM cytology and that HPV66 was equally present in all cytological groups [18]. We also observed a high prevalence of HPV 16 and HPV18 in patients with LSIL cytology and a predominance of LR-HPV types in patients with NILM cytology.

We conclude that infection with multiple HPV types is frequent in our population and that there is an association of some HPV types with cytological results.

\section{References}

1. De Roda Husman A., Walboomers J.M.M., Van Den Brule A.J.C., et al. The use of general primers GP5 and GP6 elongated at their 3' ends with adjacent highly conserved sequences improves human papillomavirus detection by PCR. J Gen Virol 1995;76(4):1057-62.

2. Manos M.M., Waldman J., Zhang T.Y., et al. Epidemiology and partial nucleotide sequence of four novel genital human papillomaviruses. J Infect Dis 1994;170(2):1096-9.

3. Strauss S., Jordens J.Z., Desselberger U., Gray J.J. Single-tube real-time nested polymerase chain reaction for detecting human papillomavirus DNA. Diagn Mol Pathol 2000;9(3):151-7.

4. Fuessel Haws A.L., He Q., Rady P.L., et al. Nested PCR with the PGMY09/11 and $\mathrm{GP}^{+} / 6^{+}$primers sets improves detection of HPV DNA in cervical samples. J Virol Methods 2004;122(1):87-93.
5. Lungu O., Wright T.CJr., Silverstein S. Typing of human papillomavirus by polimerase chain reaction amplification with L1 consensus primers and RFLP analysis. Mol. Cell. Probes 1992;6(2):145-52.

6. Meyer T., Arndt R., Stockfleth E., et al.. Strategy for typing human papillomaviruses by RFLP analysis of PCR products and subsequent hybridization with generic probe. Biotechniques 1995; 19(4):632-9.

7. Kado S., Kawamata Y., Shino Y., et al. Detection of human papillomaviruses in cervical neoplasias using multiple sets of generic polymerase chain reaction primers. Gynecol Oncol 2001;81(1):47-52.

8. Solomon D, Davey D, Kurman R, et al. The 2001 Bethesda System: terminology for reporting results of cervical cytology. JAMA 2002;287(16):2114-9.

9. Walboomers J.M., Jacobs M.V., Manos M.M., et al. Human Papillomavirus is a necessary cause of invasive cervical cancer worldwide. J Pathol 1999;189(1):12-9.

10. Huang L.W., Chao S.L., Chen P., Chou H. Multiple HPV genotypes in cervical carcinomas: improved DNA detection and typing in archival tissues. J Clin Virol 2004;29(4):271-6.

11. Munoz N., Bosch F.X., de Sanjose S., et al.. Epidemiological classification of human papillomavirus types associated with cervical cancer. N Engl J Med 2003;348(6):518-27.

12. Eluf-Neto J., Booth M., Muñoz N., et al. Human papillomavirus and invasive cervical cancer in Brazil. Br J Cancer 1994;69(1):114-9.

13. Levi J.E., Kleter B., Quint W.G.V., et al. High prevalence of Human Papillomavirus (HPV) infections and high frequency of multiple HPV genotypes in Human Immunodeficiency Virus-infected women in Brazil. J Clin Microbiol 2002;40(9):3341-5.

14. Tawheed A.R., Beaudenon S., Favre M., Orth G. Characterization of Human Papillomavirus Type 66 from an invasive carcinoma of the uterine cervix. J Clin Microbiol 1991;29(11):2656-60.

15. Matsukura T., Sugase M. Relationships between 80 human papillomavirus genotypes and different grades of cervical intraepithelial neoplasia: association and causality. Virology 2001;283(1):139-47.

16. International Agency for Research on Cancer. IARC monographs on Carcinogenicity of human papillomaviruses. Lyon, France. International Agency for Research on Cancer, 2005.

17. Poljak M., Marin I.J., Seme K., Vince A. Hybrid Capture II HPV Test detects at least 15 human papillomavirus genotypes not included in its current high-risk probe cocktail. J Clin Virol 2002;25(3):S89-S7.

18. Van Den Brule A.J.C., Pol R., Fransen-Daalmeijer N., et al. GP5+/6+ PCR followed by reverse line blot analysis enables rapid and high-throughput identification of Human Papillomavirus genotypes. J Clin Micorbiol 2002;40(3):77987.

19. Speich N., Schmitt C., Bollmann R., Bollmann M. Human Papillomavirus (HPV) study of 2916 cytological samples by PCR and DNA sequencing: genotype spectrum of patients from the west German area. J Med Microbiol 2004;53(2):1258.

20. Levi J.E., Fernandes S., Tateno A.F., et al. Presence of multiple human papillomavirus types in cervical samples from HIVinfected women. Gynecol Oncol 2004;92(1):225-31. 